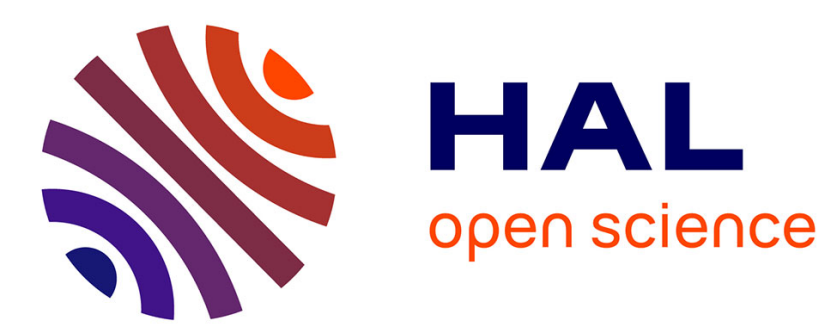

\title{
Simplification des prémesures d'événements de clichés de chambre à bulles pour le traitement au H.P.D
}

\author{
G. Pichon, C. de La Vaissière, T.P. Yiou
}

\section{To cite this version:}

G. Pichon, C. de La Vaissière, T.P. Yiou. Simplification des prémesures d'événements de clichés de chambre à bulles pour le traitement au H.P.D. Revue de Physique Appliquée, 1969, 4 (2), pp.317-318. 10.1051/rphysap:0196900402031701 . jpa-00243280

\section{HAL Id: jpa-00243280 https://hal.science/jpa-00243280}

Submitted on 1 Jan 1969

HAL is a multi-disciplinary open access archive for the deposit and dissemination of scientific research documents, whether they are published or not. The documents may come from teaching and research institutions in France or abroad, or from public or private research centers.
L'archive ouverte pluridisciplinaire HAL, est destinée au dépôt et à la diffusion de documents scientifiques de niveau recherche, publiés ou non, émanant des établissements d'enseignement et de recherche français ou étrangers, des laboratoires publics ou privés. 


\title{
SIMPLIFICATION DES PRÉMESURES D'ÉVÉNEMENTS DE GLIGHÉS DE GHAMBRE A BULlES POUR LE TRAITEMENT AU H.P.D.
}

\author{
G. PICHON, G. DE LA VAISSIÈRE et T. P. YIOU, \\ Institut de Physique Nucléaire, Paris.
}

\begin{abstract}
Résumé. - Le but est de rechercher la simplification des prémesures nécessaires à l'utilisation des appareils automatiques de clichés de chambre à bulles. La méthode consiste à ne prémesurer que sur deux vues stéréoscopiques et de trouver le masque de la troisième vue par une reconstruction géométrique. Le principe consiste à utiliser la configuration géométrique de prise de vue des clichés de la chambre à bulles. Les résultats obtenus pour la chambre à bulles de $2 \mathrm{~m}$ du C.E.R.N. et la validité de cette méthode sont discutés.

Abstract. - The purpose of this work is to simplify the premeasurements necessary for treatment bubble chamber pictures by the H.P.D. The method consists in premeasuring only on two stereoscopic views and in determining the third one by a geometrical calculation. The principle consists in using the geometrical configuration of the cameras of the bubble chamber. The results obtained for the C.E.R.N. $2 \mathrm{~m} \mathrm{H.B.C.} \mathrm{as} \mathrm{well} \mathrm{as} \mathrm{the} \mathrm{validity} \mathrm{of} \mathrm{the}$ proposed method are discussed.
\end{abstract}

Actuellement, les événements se produisant dans la chambre à bulles et destinés à être traités par les appareils de mesure automatique du type H.P.D. sont prémesurés sur trois vues stéréoscopiques. Le principal but de la prémesure est d'alléger la recherche des traces à partir de l'image digitalisée fournie par le H.P.D. Elle permet de définir des routes dans lesquelles doivent être recherchées les traces d'un événement, et ne nécessite donc pas une très grande précision. G'est ce qui nous a amenés à penser qu'il serait peut-être possible de prémesurer seulement deux vues stéréoscopiques, la troisième étant reconstruite à l'aide des deux autres par un procédé géométrique. Lorsqu'on connaît la disposition exacte des pieds optiques des caméras de prise de vue, il est possible de retrouver, à partir de deux vues, les poirts correspo:dants sur la troisième. Ce principe est à la base de la méthode de mesure graphique des clichés de chambre à bulles [1].

Notre procédé de prémesure sur deux vues est le suivant : sur chacune des deux vues, nous mesurons deux « croix de prémesure » et trois points bien répartis sur chaque trace secondaire, le premier étant toujours l'apex de l'événement. Pour reconstruire la trace correspondante sur la troisième vue, on procède de la façon suivante : 1) on choisit une vue de référence; 2) on superpose les deux « croix de prémesure » de la deuxième vue sur celles de la vue de référer:ce; 3) après cette opération, tout couple de points correspondants sur les deux vues prémesurées donre systématiquement un point correspondant sur la troisième 
vue par la méthode graphique; 4) la trace reconstruite est alors représentée par l'arc de cercle qui passe par les trois points de la troisième vue correspondant à ceux prémesurés sur la vue de référence.

La figure 1 montre la reconstruction d'un événement à 4 branches; les courbes en traits pleins représentent les traces prémesurées, tandis que les courbes pointillées représentent les mêmes traces reconstruites à partir de la méthode précédente. On voit que les résultats obtenus sont satisfaisants.

La figure 2 donne une idée générale de l'écart entre la trace mesurée et la trace reconstruite. On voit que l'écart est tout à fait acceptable pour le masque;

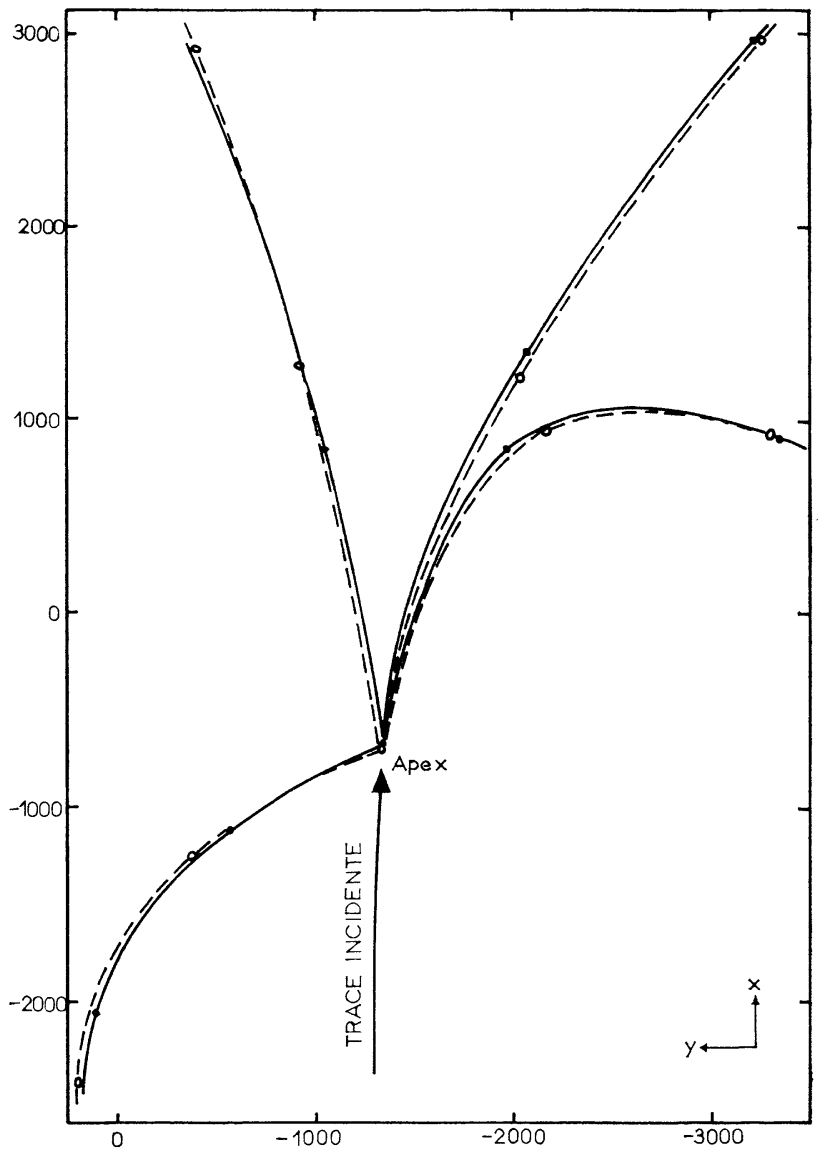

FIG. 1. - Comparaison entre les traces prémesurées d'une vue et les mêmes traces reconstruites à partir de deux autres vues. La figure représente un événement à 4 branches de l'expérience antiproton-proton à 3,6 MeV/c (C.B.H. de $2 \mathrm{~m}$ du C.E.R.N.) : — Traces prémesurées; - - Traces reconstruites. Les coordonnées sont exprimées en unités de codeur ( 1 unité de codeur correspond à $7 \mu \mathrm{m}$ sur film).

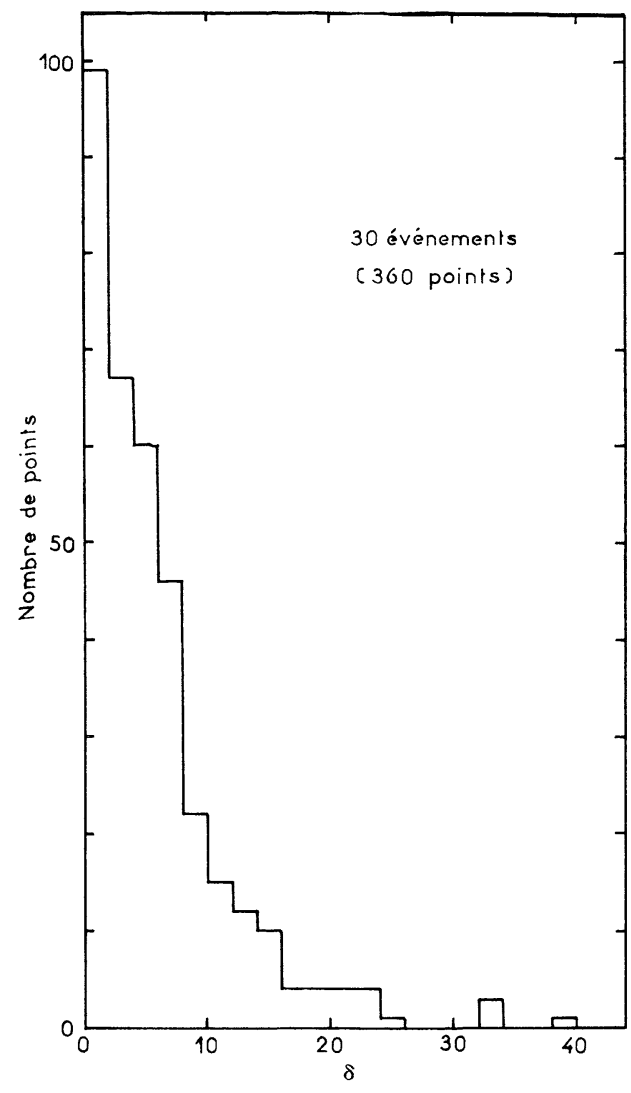

Frg. 2. - Distribution des distances $\delta$ entre les points prémesurés et les traces reconstruites ( $\delta$, unités de codeur ; 1 unité de codeur correspond à $7 \mu \mathrm{m}$ sur film).

l'écart maximal est inférieur à $630 \mu$ et $97 \%$ des points ont un écart inférieur à $175 \mu$ (l'erreur longitudinale admise pour le masque est de l'ordre de $\pm 1000 \mu$ sur le film).

Quelques centaines d'événements de l'expérience antiproton-proton à $3,6 \mathrm{GeV} / \mathrm{c}$ à haute statistique ainsi traités ont été mesurés par le H.P.D. de façon tout à fait satisfaisante.

Nous pensons que cette méthode est utilisable; elle nous permet de : 1) gagner un facteur $1 / 3$ sur le temps de prémesure $(4 \mathrm{mn}$ par événement au lieu de $6 \mathrm{mn}$ par événement); 2) simplifier la construction de la table de prémesure, ou en l'état actuel des choses utiliser deux fois moins de tables de prémesure. Mais elle nécessite un traitement de calcul supplémentaire : par exemple, sur la « GDC $3600 »$, un événement prémesuré sur trois vues demande 0,25 s/évt, alors qu'un événement prémesuré sur deux vues demande 0,54 s/évt, ce qui est négligeable.

\section{BIBLIOGRAPHIE}

[1] BoReli, (V.) et al., Nuovo Cim., 1958, 10, 525. 